\title{
Pushing absorption of perovskite nanocrystals into the infrared
}

Prachi Rastogi, ${ }^{1 \$}$ Audrey Chu ${ }^{1}$, Charlie Gréboval ${ }^{1}$, Junling Qu ${ }^{1}$, Ulrich Nguétchuissi Noumbé2

Sang-Soo Chee ${ }^{1}$, Mayank Goyal ${ }^{1}$, Adrien Khalilii', Xiang Zhen $\mathrm{Xu}^{3}$, Hervé Cruguel ${ }^{1}$, Sandrine Ithurria ${ }^{3}$, Bruno Gallas ${ }^{1}$, Jean-Francois Dayen ${ }^{2}$, Lenart Dudy ${ }^{4}$, Mathieu G. Silly ${ }^{4}$, Gilles Patriarche ${ }^{5}$, Aloyse Degiron ${ }^{6}$, Gregory Vincent ${ }^{7}$, Emmanuel Lhuillier ${ }^{{ }^{*}}$

${ }^{1}$ Sorbonne Université, CNRS, Institut des NanoSciences de Paris, INSP, F-75005 Paris, France.

${ }^{2}$ Université de Strasbourg, IPCMS-CMRS UMR 7504, 23 Rue du Loess, 67034 Strasbourg, France.

${ }^{3}$ Laboratoire de Physique et d'Etude des Matériaux, ESPCI-Paris, PSL Research University, Sorbonne Université Univ Paris 06, CNRS UMR 8213, 10 rue Vauquelin 75005 Paris, France. ${ }^{4}$ Synchrotron-SOLEIL, Saint-Aubin, BP48, F91192 Gif sur Yvette Cedex, France.

${ }^{5}$ Centre de Nanosciences et de Nanotechnologies, CNRS, University of Paris-Sud, Université Paris-Saclay, C2N, Marcoussis 91460, France.

${ }^{6}$ Université de Paris, CNRS, Laboratoire Matériaux et Phénomènes Quantiques, 75013 Paris, France.

${ }^{7}$ ONERA - The French Aerospace Lab, 6, chemin de la Vauve aux Granges, BP 80100, F-91123 Palaiseau, France.

\$ These two authors have equal contributions.

To whom correspondence should be sent: el@insp.upmc.fr

\section{Table of content}

1. MATERIALS

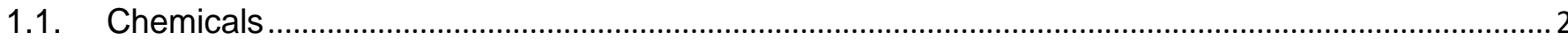

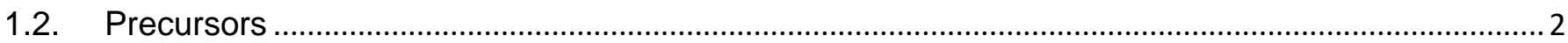

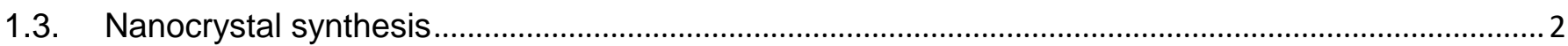

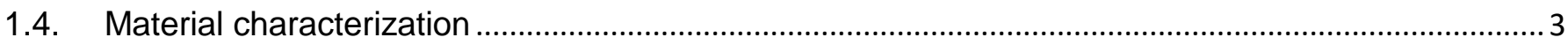

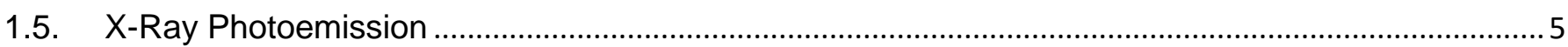

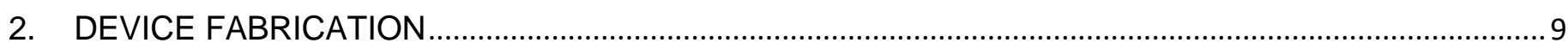

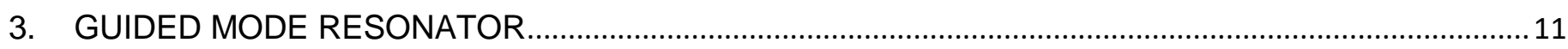

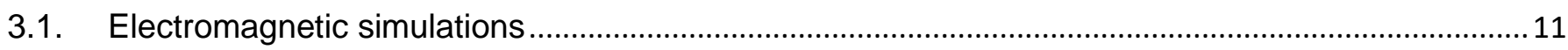

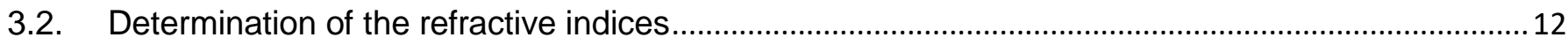

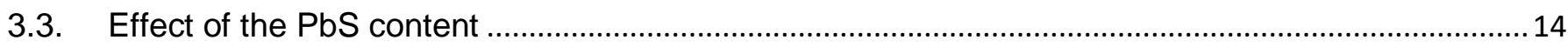

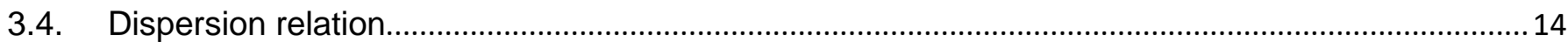

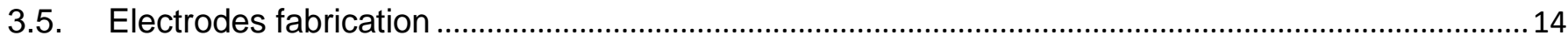

3.6. Photoluminescence characterization of the GMR electrode

3.7. Additional characterization of the FAPI/PbS film deposited onto GMR electrode............................17

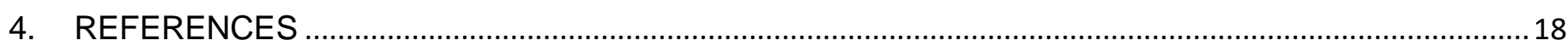




\section{MATERIALS}

\subsection{Chemicals}

\section{Chemicals for nanocrystal synthesis}

$\mathrm{Pbl}_{2}$ (Alfa Aesar, 98.5\%), formamidinium acetate ( $\mathrm{Fa}(\mathrm{OAC})_{2}$, Alfa Aesar, 99\%), oleylamine (OLA, SigmaAldrich, 80-90\%), oleic acid (OA, Sigma-Aldrich), n-hexane (VWR, 99\%), lead chloride ( $\mathrm{PbCl}_{2}$, Afla Aesar, 99\%), sulfur powder (S, Afla Aesar, 99.5\%), octadecene (ODE, Acros Organics, 90\%), lead acetate $\left(\mathrm{Pb}(\mathrm{OAc})_{2} .2 \mathrm{~Pb}(\mathrm{OH})\right.$, Alfa Aesar), ethyl acetate $\left(\mathrm{Et}(\mathrm{OAc})_{2}\right.$, Carlo Erba), ethanol absolute anhydrous (VWR), methanol (Carlo Erba, 99.8\%), toluene (Carlo Erba, 99.3\%).

\subsection{Precursors}

Preparation of stock solution of formamidinium oleate. In a $50 \mathrm{~mL}$ three-neck flask $392 \mathrm{mg}$ of formamidinium acetate with $18 \mathrm{~mL}$ of ODE and $12 \mathrm{~mL}$ of $\mathrm{OA}$ were mixed. The solution is degassed a $50{ }^{\circ} \mathrm{C}$ for $30 \mathrm{~min}$. Then the temperature was increased to $100^{\circ} \mathrm{C}$ and under $\mathrm{N}_{2}$. Once the FA-acetate was reacted to form a clear solution, the solution cooled down.

\subsection{Nanocrystal synthesis}

PbS CQD Synthesis. In a three-necked flask, $300 \mathrm{mg}$ of $\mathrm{PbCl}_{2}$ and $7.5 \mathrm{~mL}$ of OLA are degassed, first at room temperature and then at $110^{\circ} \mathrm{C}$ for $30 \mathrm{~min}$. Meanwhile $30 \mathrm{mg}$ of $\mathrm{S}$ powder is mixed with $7.5 \mathrm{~mL}$ of OLA until full dissolution and an orange clear solution is obtained. Then under $\mathrm{N}_{2}$ at $160{ }^{\circ} \mathrm{C}$, this solution of $\mathrm{S}$ is quickly added to the flask. After $15 \mathrm{~min}$, the reaction was quickly quenched by the addition of $1 \mathrm{~mL}$ of oleic acid and $10 \mathrm{~mL}$ of hexane. The nanocrystals were precipitated with ethanol and redispersed in toluene. This washing step was repeated one more time. The solution was then centrifuged to remove the unstable phase. The supernatant precipitated with methanol and redispersed in toluene. Finally, the PbS CQD solution in toluene filtered through a $0.2 \mu \mathrm{m}$ PTFE filter.

FAPI nanocrystal synthesis. In a $100 \mathrm{~mL}$ three-neck flask, $240 \mathrm{mg}$ of $\mathrm{Pbl}_{2}$ are added in $18 \mathrm{~mL}$ of ODE and degassed at $110^{\circ} \mathrm{C}$ under vacuum. After $20 \mathrm{~min}$ of degassing $2 \mathrm{~mL}$ of OLA were added. Once vacuum has recovered, $4 \mathrm{~mL}$ of $\mathrm{OA}$ was added. At this point, the lead salt was fully dissolved and the solution turns clear yellow. The atmosphere is switched to $\mathrm{N}_{2}$ and the temperature set at $80{ }^{\circ} \mathrm{C}$. Under $\mathrm{N}_{2} 20 \mathrm{~mL}$ of the formamidinium oleate is quickly injected. The solution turns dark red. After $15 \mathrm{~s}$ the heating mantle is removed and a water bath is used to cool the flask. The solution was centrifuged at $6000 \mathrm{rpm}$. The supernatant was discarded and the formed pellet redispersed in hexane. Ethyl acetate was added to precipitate the nanocrystals the second time. After centrifugation, the pellet was dispersed in toluene. 


\subsection{Material characterization}

UV-Vis spectroscopy UV-Vis spectrum of FAPI was recorded in diluted nanocrystal in hexane with JASCO V-730 spectrometer.

Infrared spectroscopy was conducted using a Fisher IS50 Fourier transform Infrared spectrometer. To measure CQD absorption, we use the spectrometer in ATR configuration. A drop of CQD solution was dried on the diamond cell. The source was a white light, the beam splitter is made of $\mathrm{CaF}_{2}$ and the detector was a DTGS ATR. Spectra are typically acquired between $10000 \mathrm{~cm}^{-1}$ and $2000 \mathrm{~cm}^{-1}$ with a $4 \mathrm{~cm}^{-1}$ resolution while averaging over 32 spectra. To measure a film absorption, an extended InGaAs detector was used and the source is a white light. In case of extended SWIR device, the visible light was switched to a global source combined with a DTGS detector. Each spectrum is averaged 32 times and the resolution is set to $4 \mathrm{~cm}^{-1}$. Photocurrent spectra are acquired as the sample was biased using a Femto DLPCA 200 current amplifier which role is also to magnify the current. The signal was then fed into the FTIR acquisition board.

For TEM images. A drop of a diluted nanocrystals solution was drop-casted on a copper grid covered with an amorphous carbon film. TEM/STEM observations were made on a Titan Themis 200 microscope (FEI/ Thermo Fischer Scientific) equipped with a geometric aberration corrector on the probe. The microscope was also equipped with the "Super-X" systems for EDX analysis with a detection angle of 0.9 steradian. The observations were made at $200 \mathrm{kV}$ with a probe current of about $70 \mathrm{pA}$ and a half-angle of convergence of $17 \mathrm{mrad}$. HAADF-STEM images are acquired with a camera length of $110 \mathrm{~mm}$ (inner/outer collection angles are respectively 69 and $200 \mathrm{mrad}$ ).

For SEM pictures. A Zeiss Supra 40 scanning electron microscope is used to acquire the SEM images. The acceleration bias was set a $10 \mathrm{kV}$ and the aperture at $20 \mu \mathrm{m}$.

EDX analysis. Chemical analysis of the sample has been conducted using Energy dispersive X-ray spectroscopy (EDX) either at the film level, see Figure $S 1$ and Table $S 1$ or at the nanoparticle level, see Figure $S 2$. Both analyses confirm the presence of the two materials in the mixture. EDX coupled to STEM confirmed that there is no phase segregation.

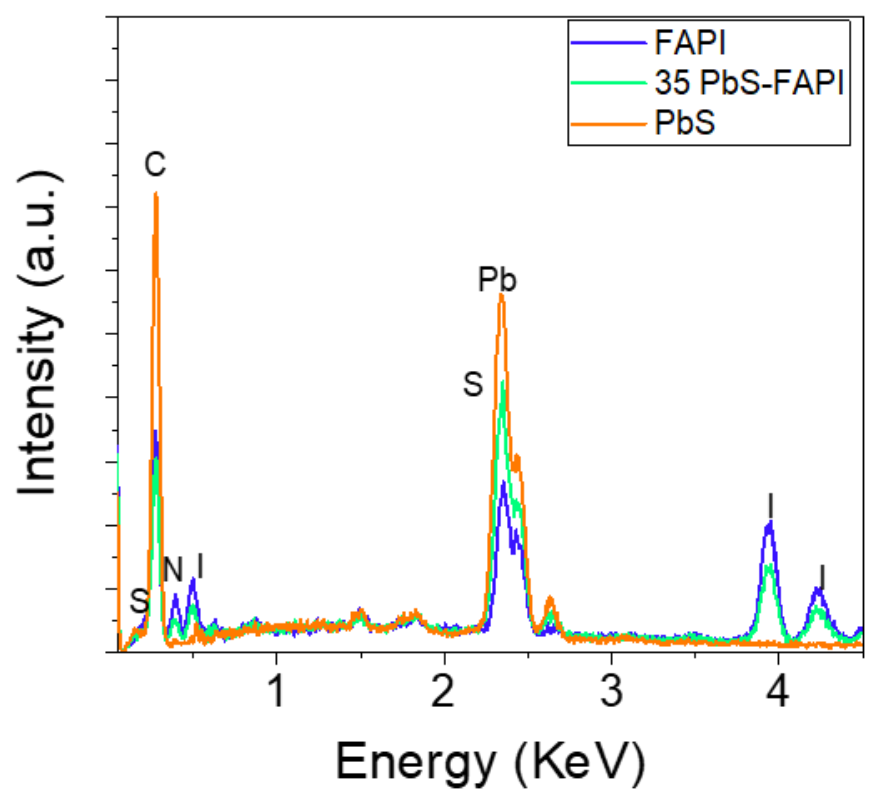

Figure $S 1$ EDX spectrum relative to FAPI, PbS and FAPI/PbS mixture. 
Table $S 1$ Ratio of $\mathrm{Pb}$ to $\mathrm{S}$ and $\mathrm{Pb}$ to I in FAPI, PbS and FAPI/PbS mixture.

\begin{tabular}{|l|l|l|l|}
\hline Atomic \% & FAPI & $\begin{array}{l}35 \% \text { PbS- } \\
\text { FAPI }\end{array}$ & PbS \\
\hline $\mathrm{Pb} / \mathrm{S}$ & - & 2.5 & 1.35 \\
\hline $\mathrm{Pb} / \mathrm{l}$ & 0.3 & 0.65 & - \\
\hline
\end{tabular}

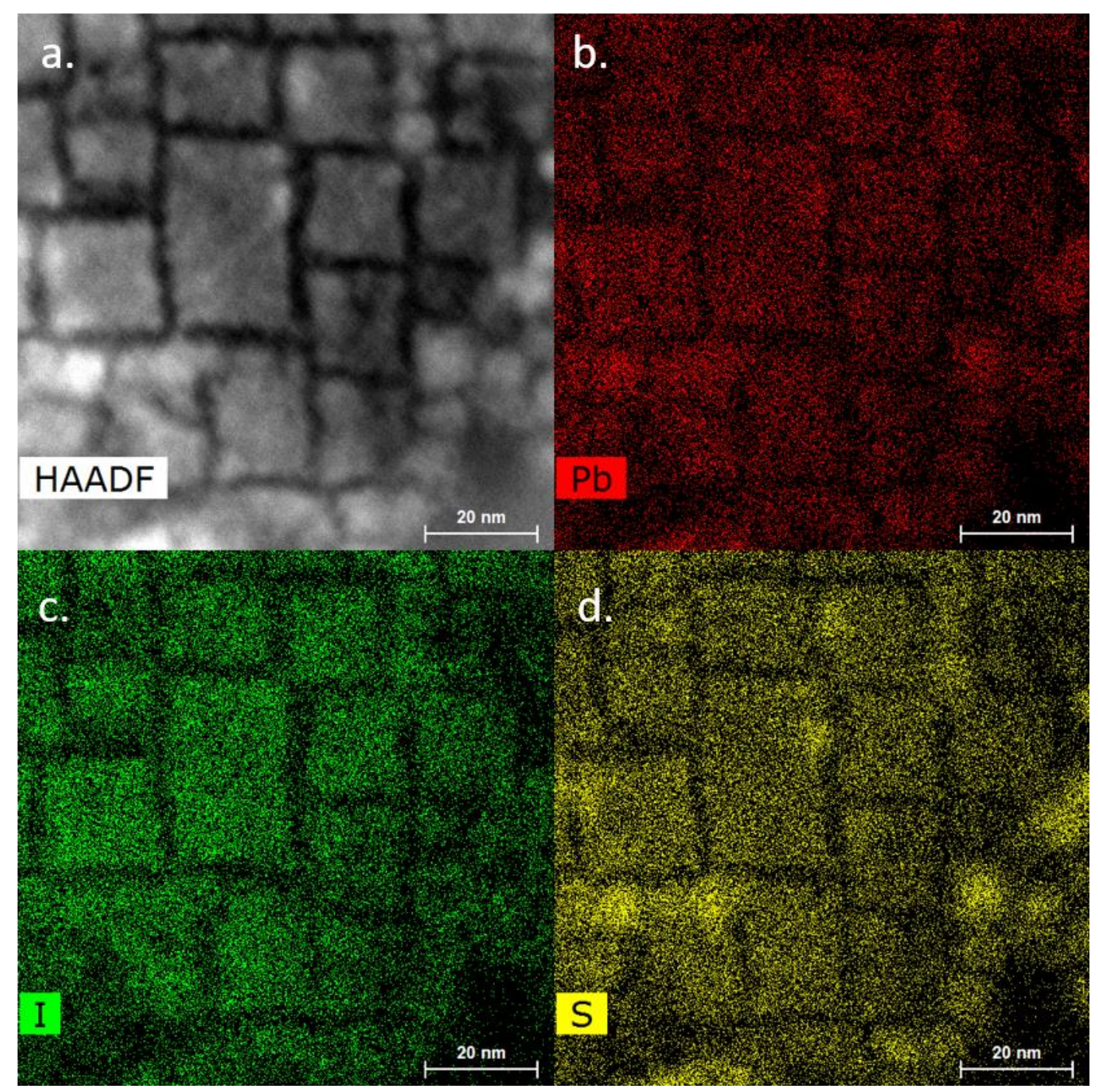

Figure S 2 STEM image from FAPI/PbS mixture (35\% PbS content): a. High angle annular dark field image, b. Intensity of the EDX relative to $\mathrm{Pb}$. $\mathrm{c}$. Intensity of the EDX relative to $\mathrm{l}$. $\mathrm{d}$. Intensity of the EDX relative to S. 


\subsection{X-Ray Photoemission}

X-ray photoemission measurements (XPS): For photoemission spectroscopy, we use the Tempo beamline of synchrotron Soleil. Films of nanocrystals were spin-casted on a gold coated Si substrate (gold layer is 80 $\mathrm{nm}$ thick). The ligands of the nanocrystals were exchanged using the same procedure as for transport measurement to avoid any charging effect during measurements. Samples were introduced in the preparation chamber and degassed until vacuum below $10^{-9} \mathrm{mbar}$ is reached. Then samples were introduced in the analysis chamber. The signal was acquired onto a MBS A-1 photoelectron analyser equipped with a delay line detector developed by Elettra. ${ }^{1}$ Acquisition was done at constant pass energy $(50 \mathrm{eV})$ within the detector. Photons energy of $150 \mathrm{eV}$ was used for acquisition of valence band and work function while a $600 \mathrm{eV}$ photon energy is used for the analysis of the core level

Calibration procedure: A gold substrate was used to calibrate the Fermi energy. The absolute value of the incoming photon energy is determined by measuring the second order of Au core level peaks. From this calibrated energy scale, we measure the energy of the Au $4 \mathrm{f}$ from the gold on the manipulator. This will later serve as reference to measure the exact photon energy every time the incident photon energy is tuned. Note that work function we apply an $18 \mathrm{~V}$ bias, which exact value has been determined by looking at the shift of a Fermi edge.

Core level analysis: The photoemission data are given in Figure $S$ 3and Figure $S 4$ for pristine FAPI, in Figure $\mathrm{S} 5$ and Figure $\mathrm{S} 6$ for pristine $\mathrm{PbS}$ and finally in Figure $\mathrm{S} 7$ and Figure $S 8$ for the hybrid FAPI/PbS (35\% PbS ratio) hybrid material. FAPI material presents contribution from the $\mathrm{Pb}, \mathrm{I}, \mathrm{N}$ and $\mathrm{C} . \mathrm{N}$ and $\mathrm{C}$ contribution result from both formamidium and ligands. $\mathrm{PbS}$ presents contribution for $\mathrm{Pb}$ and $\mathrm{S}$, as well as $\mathrm{Cl}$ from the $\mathrm{PbCl}_{2}$ used as precursor. I and $\mathrm{S}$ contribution are reasonably well fitted with a single contribution. The $\mathrm{Pb} 4 \mathrm{f}$ state systematically presents two contributions. In the case of FAPI, the binding energies are 137.3 and $138.4 \mathrm{eV}$ and can be respectively attributed to $\mathrm{Pb}^{0}$ and $\mathrm{Pb}^{2+}$. In the case of $\mathrm{PbS}$, the two contributions appear at $138.35 \mathrm{eV}$ and $139.2 \mathrm{eV}$. The first one is due to $\mathrm{Pb}^{2+}$ where $\mathrm{Pb}$ is coupled to $\mathrm{S}$ in $\mathrm{PbS}$. The one at higher binding energy is due to the presence of an oxide shell, the higher electronegativity of the oxygen as compared to the sulfur leads to the observed shift. In the hybrid material, due to the low PbS content the latter contribution is not observed. 


\section{- Photoemission signal relative to FAPI}
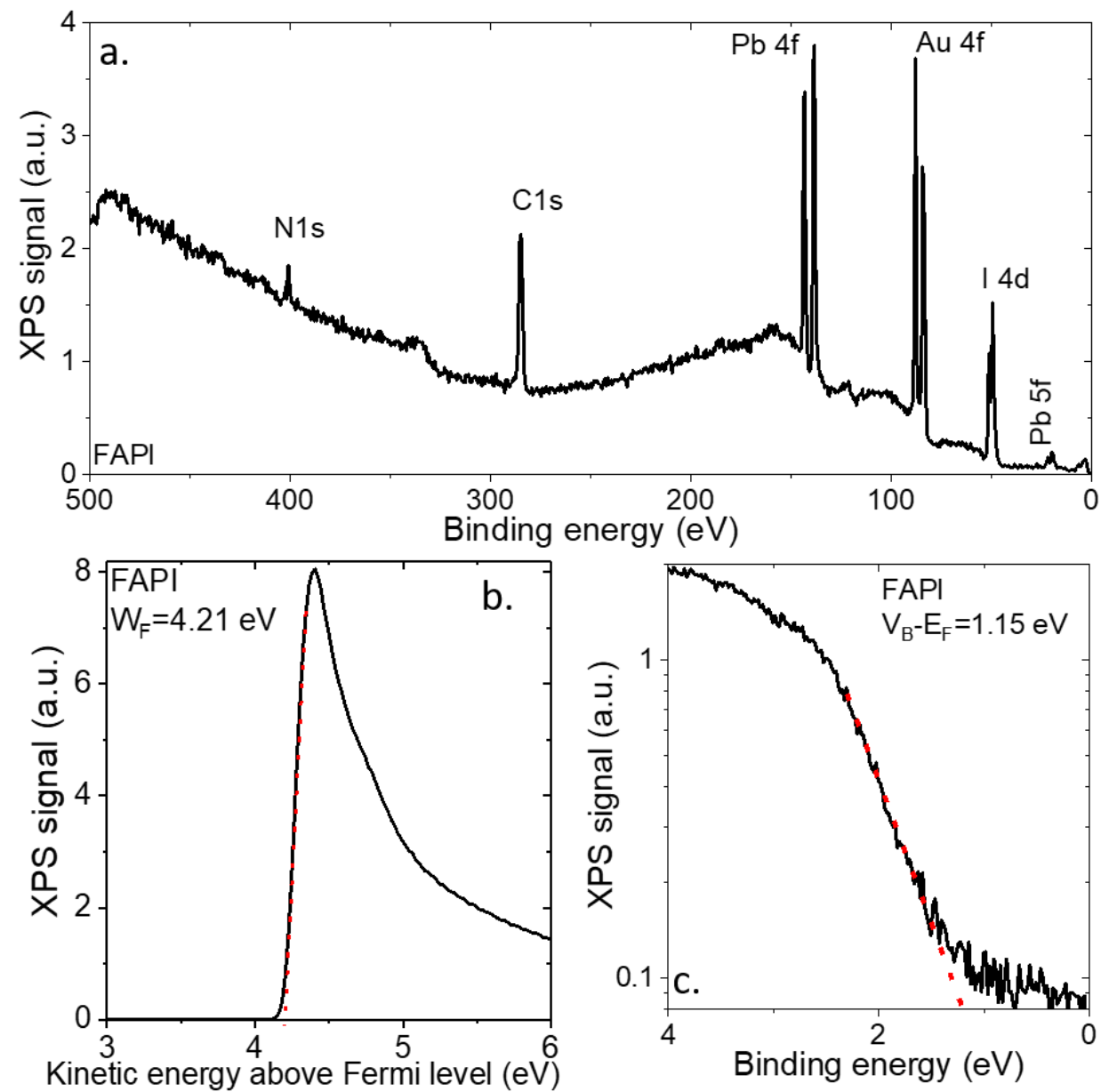

Figure S 3 a. X-ray photoemisssion overview spectrum for a thin film of FAPI nanocrystals on a gold substrate. b. X-ray photoemisssion spectrum relative to the cut-off of the secondary electron for a thin film of FAPI nanocrystals. c. X-ray photoemisssion spectrum relative to the valence band for a thin film of FAPI nanocrystals.
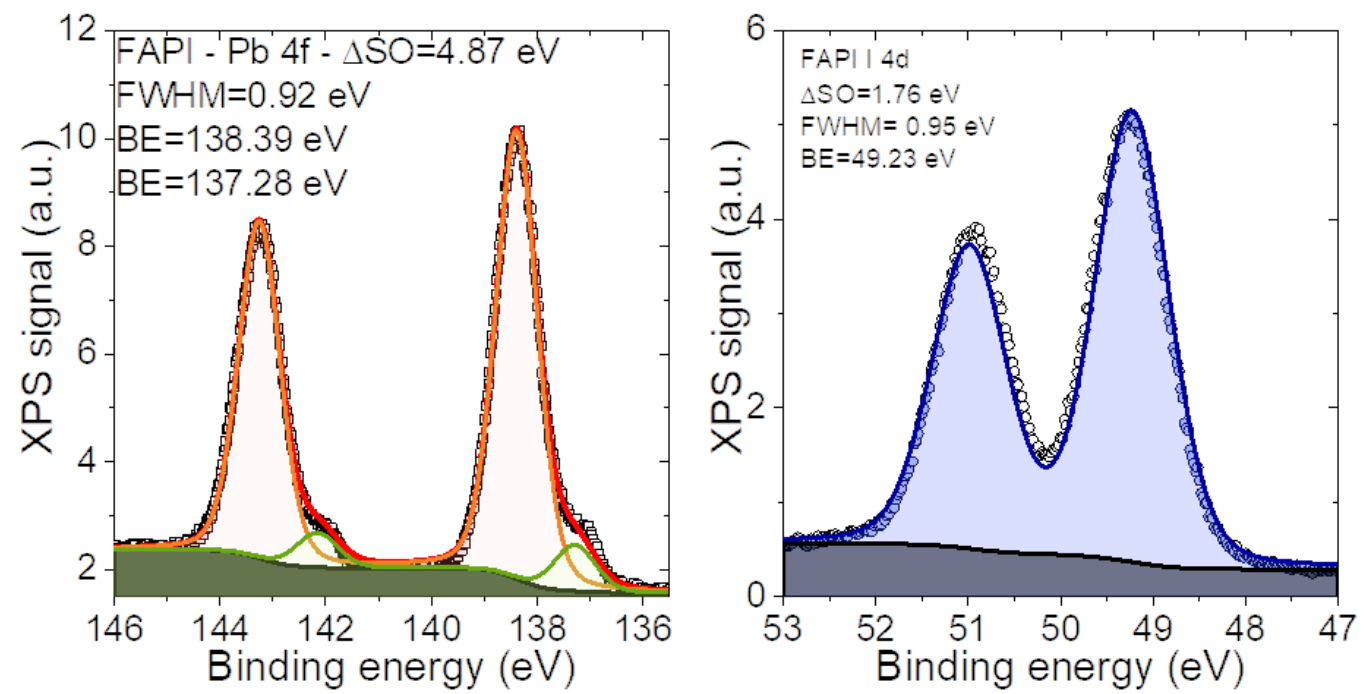

Figure $S 4$ a. X-ray photoemisssion spectrum relative to the $\mathrm{Pb} 4 f$ state for a thin film of FAPI nanocrystals. b. $X$-ray photoemisssion spectrum relative to the I $4 d$ state for a thin film of FAPI nanocrystals. 
- Photoemission signal relative to $\mathrm{PbS}$
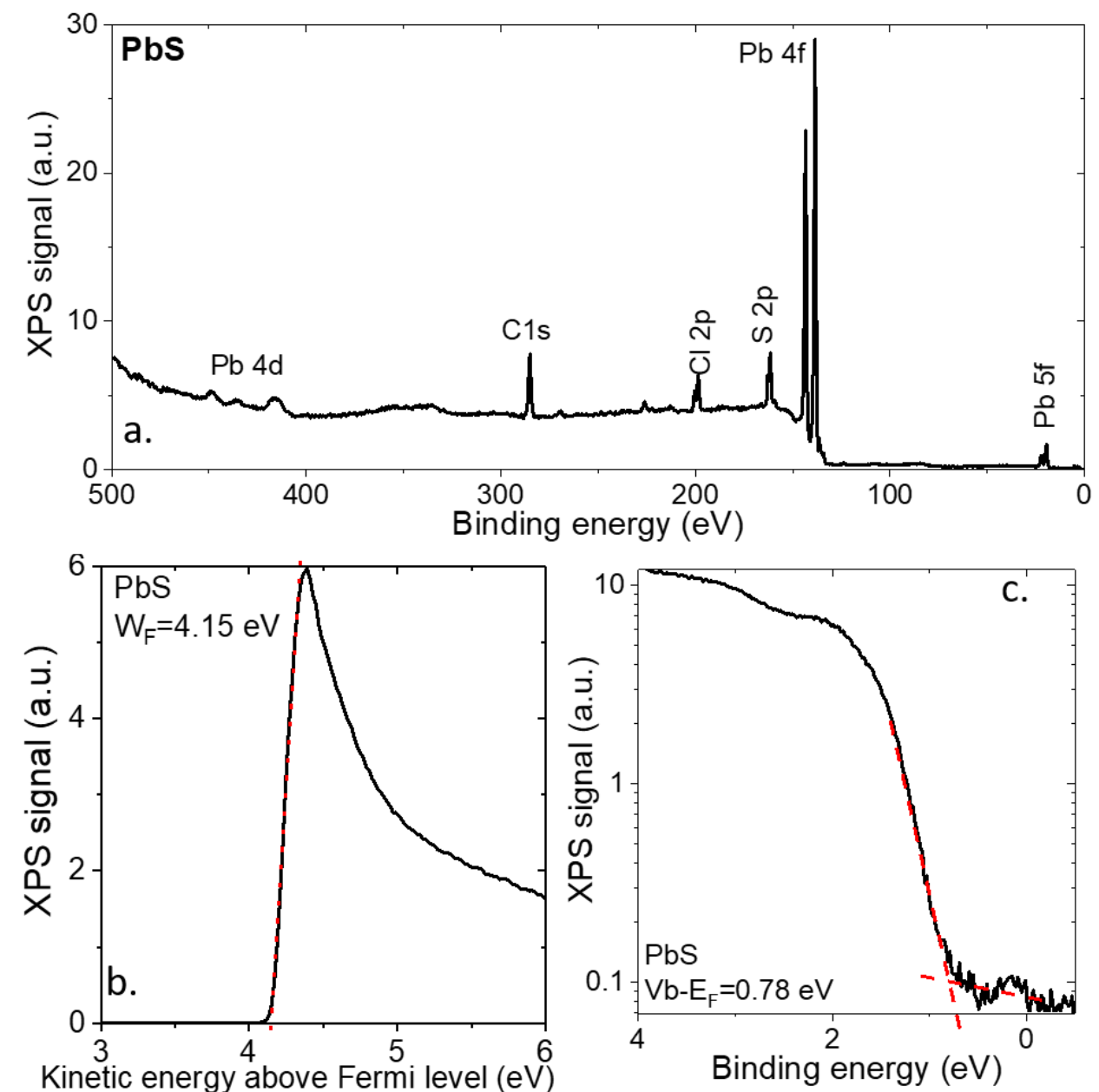

Figure $S 5$ a.X-ray photoemisssion overview spectrum for a thin film of PbS nanocrystals on a gold substrate. b. X-ray photoemisssion spectrum relative to the cut-off of the secondary electron for a thin film of PbS nanocrystals. c. X-ray photoemisssion spectrum relative to the valence band for a thin film of PbS nanocrystals.
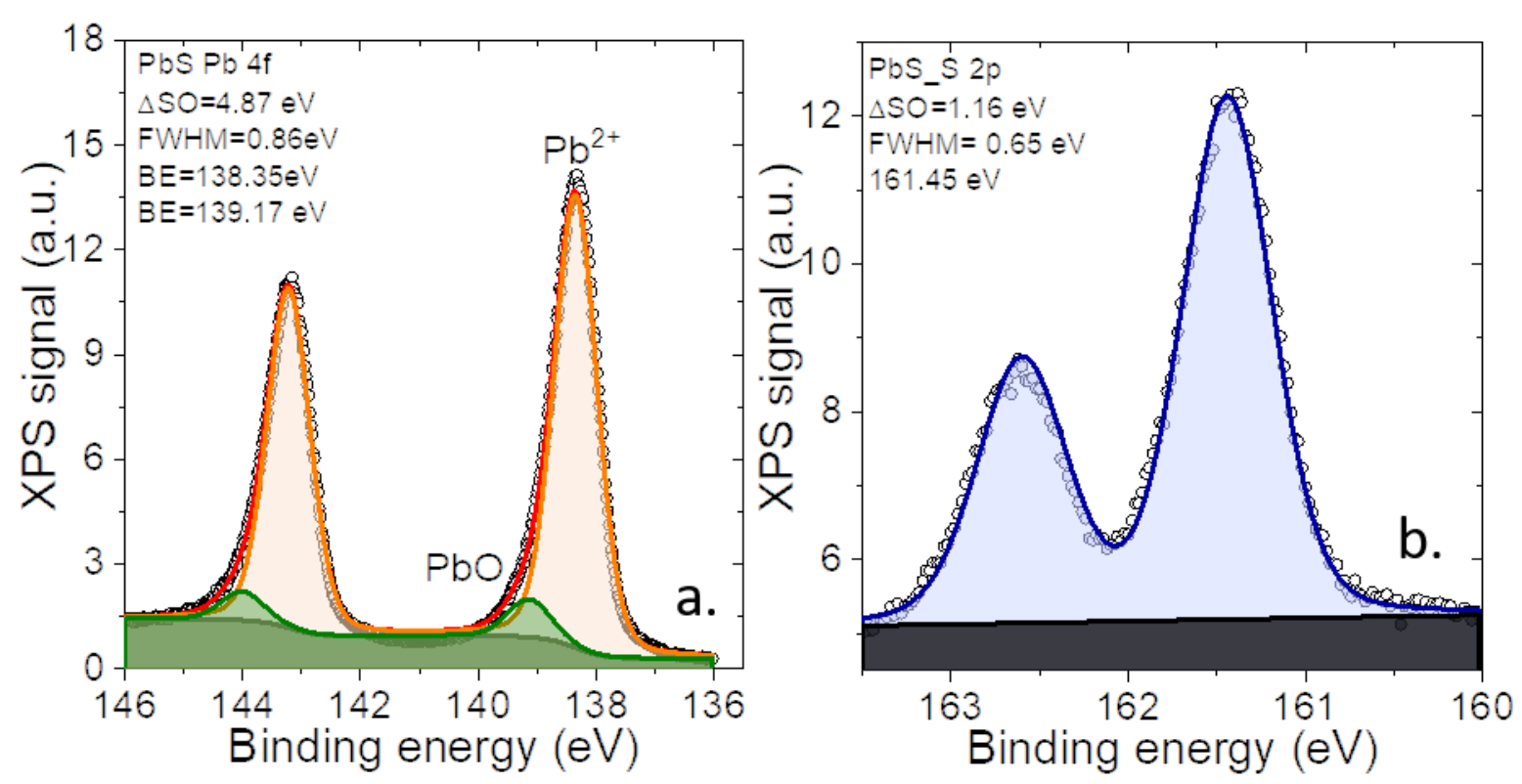

Figure $S 6$ a. X-ray photoemisssion spectrum relative to the $P b 4 f$ state for a thin film of $P b S$ nanocrystals. b. $X$-ray photoemisssion spectrum relative to the $S 2 p$ state for a thin film of PbS nanocrystals. 
- Photoemission signal relative to FAPI/PbS (35\% PbS content)
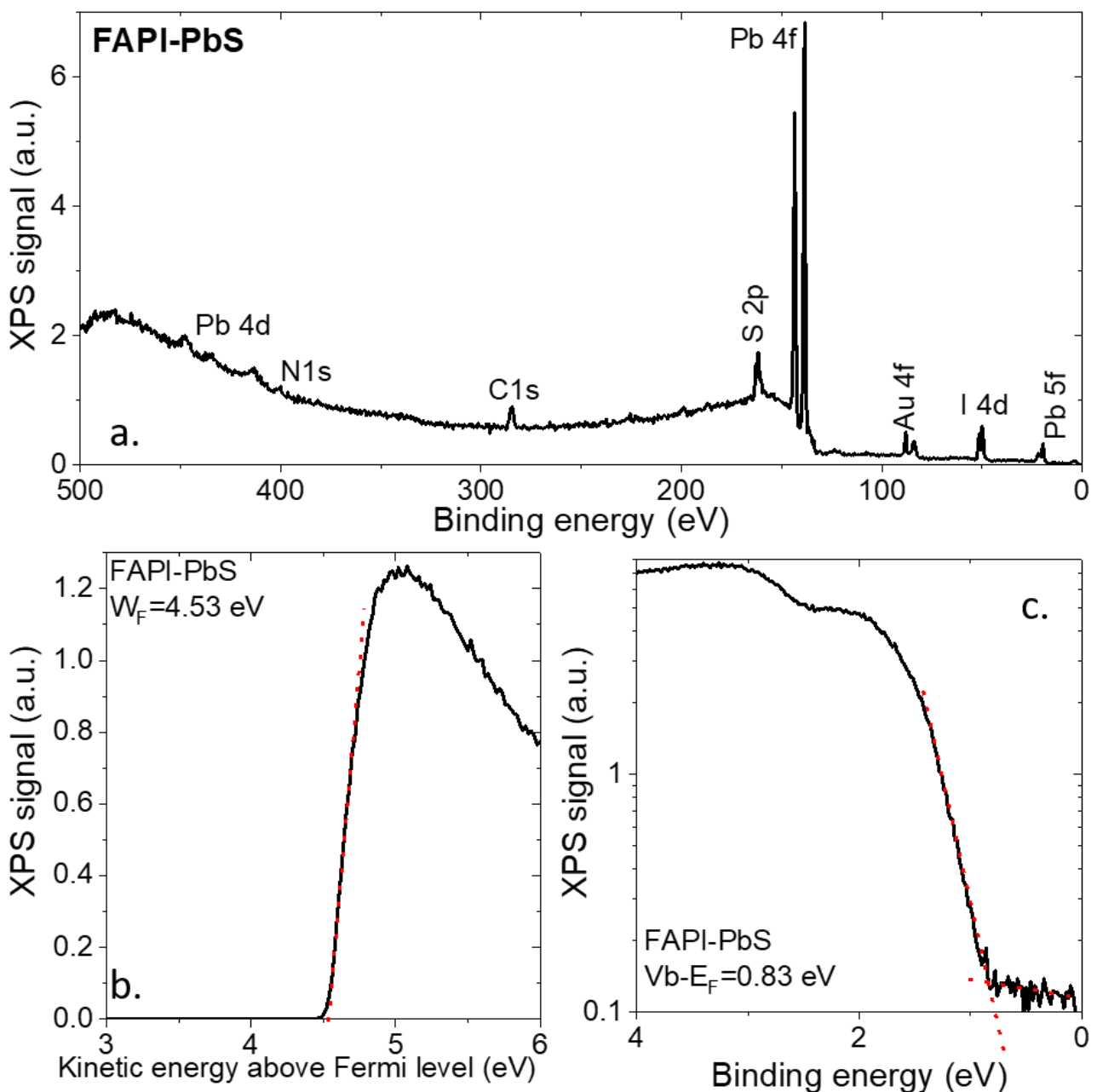

Figure $S 7 X$-ray photoemission overview spectrum for a thin film of FAPI/PbS (35\% PbS) nanocrystals on a gold substrate. $b X$-ray photoemission spectrum relative to the cut-off of the secondary electron for a thin film of FAPI/PbS (35\% PbS) nanocrystals. C. X-ray photoemission spectrum relative to the valence band for a thin film of $F A P I / P b S(35 \%$ PbS) nanocrystals.
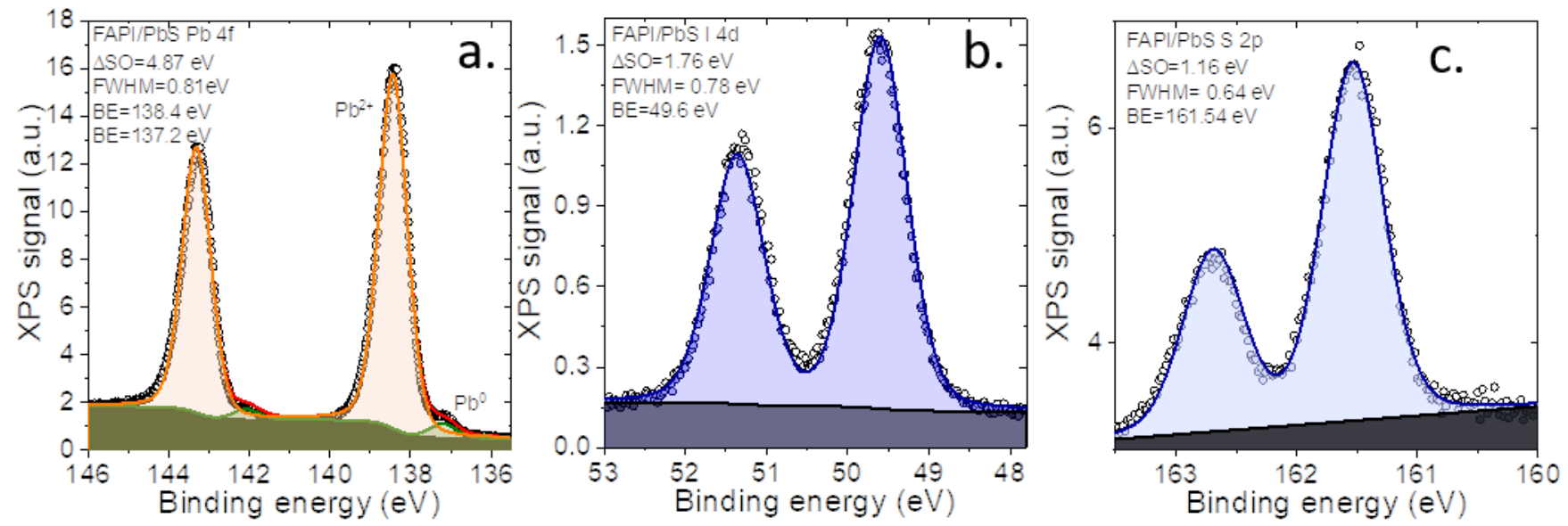

Figure $S 8$ a. X-ray photoemission spectrum relative to the $P b 4 f$ state for a thin film of FAPI/PbS (35\% PbS) nanocrystals. $b$. X-ray photoemission spectrum relative to the I $4 d$ state for a thin film of $F A P I / P b S(35 \% P b S)$ nanocrystals. $c$. $X$-ray photoemission spectrum relative to the $S 2 p$ state for a thin film of FAPI/PbS $(35 \%$ $\mathrm{PbS}$ ) nanocrystals. 


\section{DEVICE FABRICATION}

Interdigitated electrodes. The surface of $\mathrm{Si} / \mathrm{SiO}_{2}$ wafer $(400 \mathrm{~nm}$ oxide layer) is cleaned by sonication in acetone. The wafer is rinsed with acetone, then isopropanol and dried with a $\mathrm{N}_{2}$ gun. A final cleaning is made using an $\mathrm{O}_{2}$ plasma. An adhesion promoter (TI PRIME) is spin-coated onto the substrate and baked at 120 ${ }^{\circ} \mathrm{C}$ for $2 \mathrm{~min}$. AZ5214E is spin-coated and baked at $110^{\circ} \mathrm{C}$ for $90 \mathrm{~s}$. The substrate is exposed under UV through a pattern mask for $1.5 \mathrm{~s}$. The film is then baked at $125^{\circ} \mathrm{C}$ for $2 \mathrm{~min}$ in order to invert the resist. Then a $40 \mathrm{~s}$ flood exposure is performed. The resist is developed using a bath of AZ726 for $25 \mathrm{~s}$, rinsed in pure water and dried with $\mathrm{N}_{2}$. We then deposit $5 \mathrm{~nm}$ of chromium and $80 \mathrm{~nm}$ of gold using thermal evaporation. The lift-off is performed by dipping the film in acetone for 1 hour. The electrodes are rinsed using isopropanol and dried using a $\mathrm{N}_{2}$ gun. The electrodes are $2.5 \mathrm{~mm}$ long, $10 \mu \mathrm{m}$ large and spaced by $20 \mu \mathrm{m}$. These electrodes are used for electrolyte-gated transistor measurements.

Transistor on $\mathrm{LaF}_{3}$ fabrication. Commercially available $\mathrm{LaF}_{3}$ substrates have been purchased at Crystran (UK). 49 interdigits with $10 \mu \mathrm{m}$ thickness and $750 \mu \mathrm{m}$ length, spaced by $10 \mu \mathrm{m}$ were patterned by e-beam lithography using a Zeiss Supra 40 scanning electron microscope coupled to a Raith lithography system. We used a standard bilayer PMMA (Polymethyl methacrylate) resist and a conductive Protective Coating (espacer) resist to write on the insulator surface. The firsts, consisting of AR-PMMA 669.04 and AR-PMMA 679.02 , were spin-coated at 4000 revolutions per minute $(\mathrm{rpm})$ during $1 \mathrm{~min}$ and annealed at $180^{\circ} \mathrm{C}$ during $90 \mathrm{~s}$ ( $220 \mathrm{~nm} / 4000 \mathrm{rpm}$ and $70 \mathrm{~nm} / 4000 \mathrm{rpm}$ respectively). The e-spacer AR-PC 5090.02 is then spincoated at the same speed (4000 rpm) but annealed at $90{ }^{\circ} \mathrm{C}$ during $90 \mathrm{~s}(90 \mathrm{~nm} / 4000 \mathrm{rpm})$. After the development in the 1:3 MIBK-IPA at $25^{\circ} \mathrm{C}$, metal deposition $\mathrm{Ti}(3 \mathrm{~nm}) / \mathrm{Au}(57 \mathrm{~nm})$ was performed by electron beam evaporator in high vacuum chamber, followed by lift-off in acetone.

Ligand exchange procedure and thin film preparation. The ligand exchange solution was prepared by adding the $\mathrm{Pb}$-acetate powder in ethyl acetate. The undissolved $\mathrm{Pb}$-acetate was removed by centrifuging the solution. FAPI and PbS CQDs were mixed in various ratio inside a $\mathrm{N}_{2}$ filled glovebox. The FAPI/PbS nanocrystals in toluene were spin-coated at $1500 \mathrm{rpm}$ on pre-patterned interdigitated or GMR gold electrodes. After a complete drying, $\mathrm{Pb}$-acetate ligand exchanged was performed by dipping the film in Pb-acetate in ethyl acetate for $30 \mathrm{~s}$ and rinse it in pure ethyl acetate for $30 \mathrm{~s}$. The process was repeated until the desire thickness has been achieved.

Transport Measurement. The sample is connected to a Keithley 2634b, which controls the drain bias ( $\left.\mathrm{V}_{\mathrm{DS}}\right)$ and measures the associated current $\left(I_{D S}\right)$. This measure is carried out in the dark or under illumination using $1.55 \mu \mathrm{m}$ laser diode.

Transistor Measurement. The sample is connected to a Keithley 2634b, which sets the drain source bias $\left(V_{D S}\right)$, controls the gate bias $\left(V_{G S}\right)$ with a step of $1 \mathrm{mV}$ and measure the associated currents ( $\mathrm{I}_{\mathrm{DS}}$ and $\left.\mathrm{I}_{\mathrm{GS}}\right)$.

Responsivity Measurement. To measure the responsivity the photocurrent is reordered with laser diode at $1.55 \mu \mathrm{m}$. The $1.55 \mu \mathrm{m}$ laser is placed at $20 \mathrm{~cm}$ from the sample. The total spot power is measured to be 0.15 $\mathrm{mW}$. The spot size is $1.6 \mathrm{~mm}^{2}$, corresponding to irradiance of $9.4 \mathrm{~mW} . \mathrm{cm}^{-2}$. The flux is chopped form $1 \mathrm{~Hz}$ to $10 \mathrm{kHz}$. The photocurrent is measured using Zurich Instruments MFLI lock-in amplifier at $0.5 \mathrm{~V}$ bias.

Noise measurement. The sample is biased using a Femto DLPCA-200 and the flowing current (kept in the dark) is amplified by a Femto DLPCA-200, then fed into a SRS SR780 signal analyzer.

Detectivity determination. The detectivity (in Jones) of the sample is determined using the formula: $D^{*}=$ $\frac{R \sqrt{A}}{S_{I}}$, where $\mathrm{R}$ (in $\mathrm{A} \cdot \mathrm{W}^{-1}$ ) is the responsivity, $\mathrm{S}$ is the noise $(\mathrm{A} / \sqrt{\mathrm{Hz}})$ and $\mathrm{A}$ the optical area of the device $\left(\mathrm{cm}^{2}\right)$. 
Figure $S 9$ provides the time response of the hybrid FAPI/PbS deposited onto interdigitated electrodes for three content of $\mathrm{PbS}$. At low $\mathrm{PbS}$ content only a weak slow response is observed and likely have a bolometric effect. Faster response occurs when PbS is introduced.
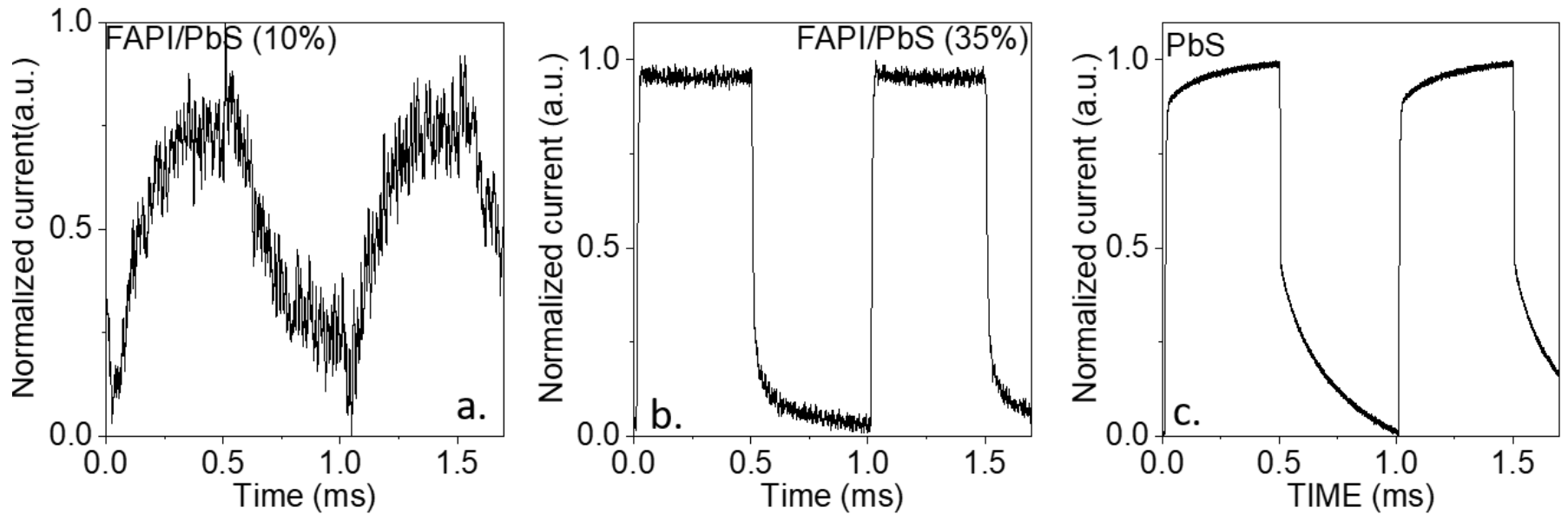

Figure $S 9$ a. (resp b.) Current as a function of time for a PbS/FAPI mixture containing $10 \%$ of PbS (resp. $35 \%$ of $\mathrm{PbS}$ ) onto interdigitated electrodes as the light $\left(\lambda=1.55 \mu \mathrm{m}-P=1.25 \mathrm{~W} . \mathrm{cm}^{-2}\right)$ is turned on and off. $\mathrm{c}$. Current as a function of time for a pure PbS film onto interdigitated electrodes as the light $(\lambda=1.55 \mu \mathrm{m}-$ $\left.P=1.25 \mathrm{~W} . \mathrm{cm}^{-2}\right)$ is turned on and off.

Figure S 10 provide full optoelectronic characterization of the FAPI/PbS (35\% PbS content) film.
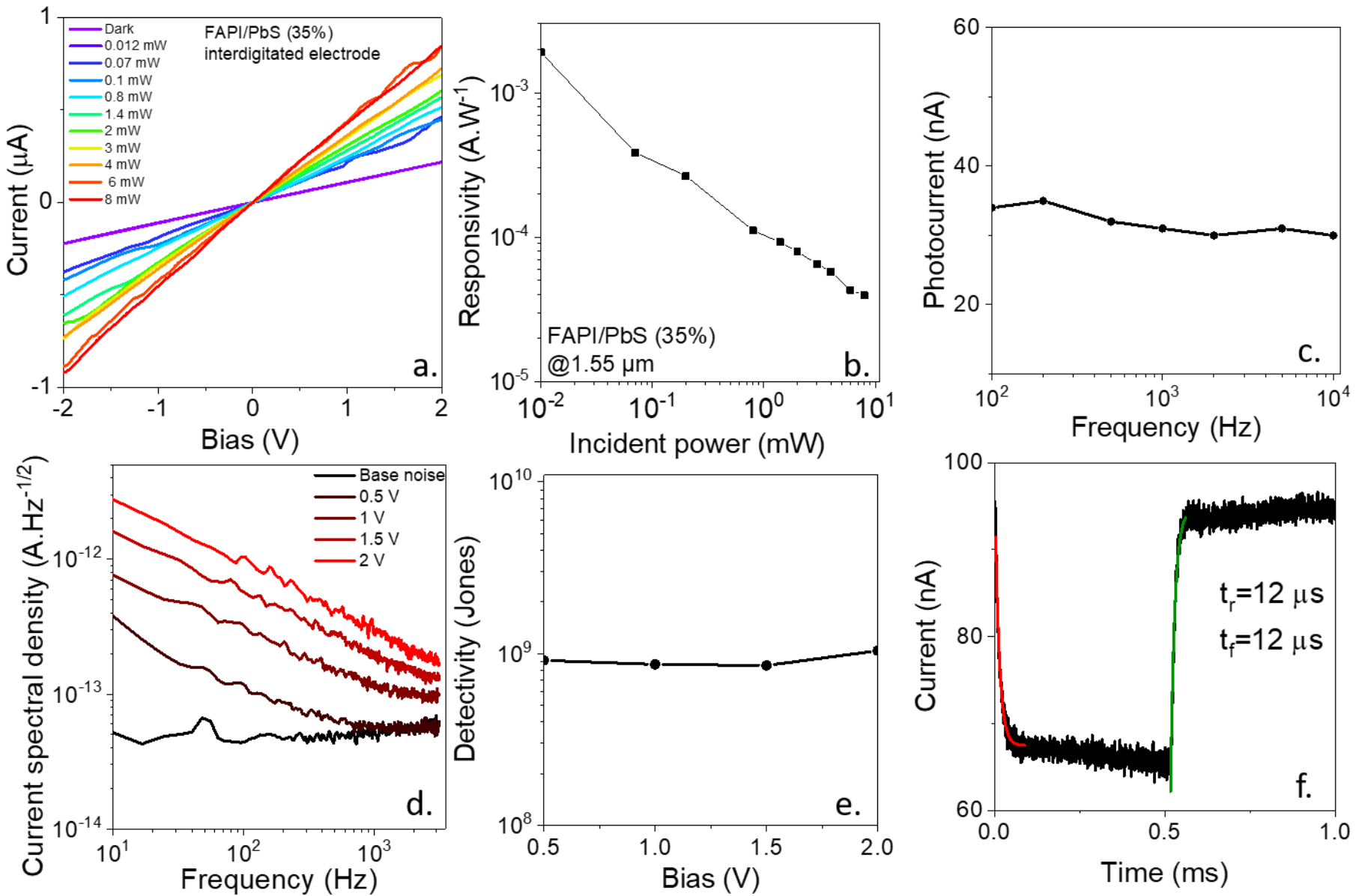

Figure S 10 a. I-V curve for the FAPI/PbS (35\% PbS content) film deposited on the interdigitated electrode under illumination by a $1.55 \mu$ m laser diode with various incident power. b. Responsivity of FAPI/PbS $(35 \%$ $\mathrm{PbS}$ content) film deposited on the interdigitated electrodes as a function of the incident power. $c$. Photocurrent of FAPI/PbS (35\% PbS content) film deposited on the interdigitated electrodes as a function of 
the signal frequency. $d$. Noise current spectral density as a function of the signal frequency for FAPI/PbS (35\% PbS content) film deposited on the interdigitated electrodes. e. detectivity as a function of the applied bias for FAPI/PbS (35\% PbS content) film deposited on the interdigitated electrodes. $f$. Current as a function of time for a PbS/FAPI mixture (35\% PbS content) onto interdigitated electrodes as the light $(\lambda=1.55 \mu \mathrm{m}-$ $\left.P=0.03 \mathrm{~W} . \mathrm{cm}^{-2}\right)$ is turned on and off.

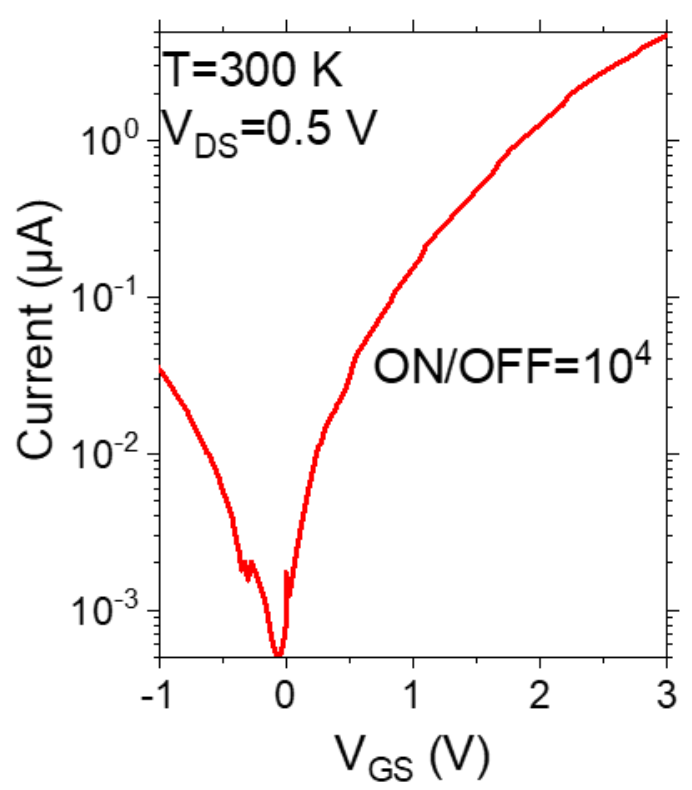

Figure $S 11$ Transfer curves (drain and gate current as a function of the applied gated bias under a constant drain source bias set equal to $0.5 \mathrm{~V}$ ) for a field effect transistor based on a LaF 3 substrate used as gate and channel made of a FAPI/PbS (35\% PbS content) nanocrystal thin film.

\section{GUIDED MODE RESONATOR}

\subsection{Electromagnetic simulations}

Calculations have been achieved with Matlab library based on Rigorous Coupled-Wave Analysis (RCWA) ${ }^{2}$. We considered incoming plane wave under normal incidence, either with transverse magnetic (TM) or transverse electric (TE) polarization, i.e. with magnetic field or electric field parallel with the slits of the gratings, respectively. Grating is supposed to be invariant along $y$ direction and repeated infinitely along $x$ direction.

For the GMR device, the layers are given in Table S2

Table S2: Layer thicknesses for each stack

\begin{tabular}{cc}
\hline Layer & Interdigitated on $\mathrm{Au} / \mathrm{SiO}_{2}$ for $\mathrm{GMR}$ \\
\hline $\begin{array}{c}\text { External } \\
\text { environment }\end{array}$ & Air \\
\hline \hline
\end{tabular}




\begin{tabular}{cc}
\hline \hline Film & $\begin{array}{c}\text { Mix PbS:FAPI } \\
\mathrm{d}=200 \mathrm{~nm}\end{array}$ \\
\hline \multirow{2}{*}{ Structure } & Mix PbS:FAPI/Au (period $\mathrm{p}$ and $\left.\mathrm{W}_{\mathrm{Au}}\right)$ \\
& $\mathrm{t}_{\mathrm{g}}=50 \mathrm{~nm}$ \\
\hline \multirow{2}{*}{ Dielectric } & $\mathrm{SiO}_{2}$ \\
& $\mathrm{LSiO} 2_{\mathrm{S}}=120 \mathrm{~nm}$ \\
\hline \multirow{2}{*}{ Gold mirror } & $\mathrm{Au}$ \\
& $200 \mathrm{~nm}$ \\
\hline \hline
\end{tabular}

The absorption map is deduced from the electric field at each point:

$$
\begin{gathered}
\text { Absorption }=\frac{\pi}{\lambda} \int_{S} \operatorname{Im}(\varepsilon(M))\left(\left|E_{y}\right|^{2}\right) d S, \quad \text { In TE polarization } \\
\text { Absorption }=\frac{\pi}{\lambda} \int_{S} \operatorname{Im}(\varepsilon(M))\left(\left|E_{x}\right|^{2}+\left|E_{z}\right|^{2}\right) d S, \quad \text { In TM polarization }
\end{gathered}
$$

\subsection{Determination of the refractive indices}

The last input needed for these calculations is the complex refractive index of each layer. For the gold ${ }^{3}$ we used previously reported refractive indices. For the $\mathrm{SiO}_{2}$, we use $\mathrm{n}=1.44$ and $\mathrm{k}=0$, and $\mathrm{n}=1$ for the air. For the imaginary part of the nanocrystals, we used the shape of the absorption feature, see Figure $\mathrm{S} 12 \mathrm{a}$. For FAPI, in the range of $1000 \mathrm{~nm}$ to $3000 \mathrm{~nm}$, the $\operatorname{Im}(\mathrm{n})=\mathrm{k}$ is set to be zero because there is no absorption. In the case of $\mathrm{PbS}$ we calculated $\operatorname{Im}(\mathrm{n})$ at the band edge using Moreel's paper ${ }^{4}$ to give an absolute value. Using (1) and (2) it is possible to calculate the absorption coefficient at the band edge and to deduce the $\operatorname{Im}(\mathrm{n})$ at that point using (3).

$$
\begin{aligned}
& E_{0}=\frac{1}{0.016 d^{2}+0.209 d+0.45} \\
& \mu=\frac{1.85 * 10}{d^{2}}\left(\mathrm{~cm}^{-1} . \mathrm{meV}\right)
\end{aligned}
$$

Where $E_{0}$ is the band edge energy, $d$ the particle diameter and $\mu$ the integrated absorption coefficient.

$$
k=\frac{\alpha \lambda}{4 \pi}
$$

With $k=\operatorname{Im}(\underline{n}), \lambda$ is the wavelength and $\alpha$ is the absorption coefficient $\left(\mathrm{cm}^{-1}\right)$.

Finally, the imaginary part of the refractive index for a NCs film (with a ratio $r$ of $\mathrm{PbS}$ ) is given by:

$$
k_{Q D S}=\mathrm{r} \cdot k_{P b S}+(1-\mathrm{r}) \cdot k_{F A P I}
$$

For the real part of the nanocrystal film refractive index, the value of 2 is commonly used for nanocrystal thin film. However, this value was in contradiction with ellipsometry measurements conducted on FAPI, see Figure $\mathrm{S} 12 \mathrm{~b}$. In order to determine the real value of the real part of the refractive index of the nanocrystal film, we simulated and designed structure ( $p$ and $w_{\mathrm{Au}}$ ) with a resonance at $1.55 \mu \mathrm{m}$ for different $\mathrm{n}$, see Table S3. Then we measured those structures and the experimental one with a resonance at $1.55 \mu \mathrm{m}$ gives us the refractive index. Experimentally, we find $n=1.6$, see Figure $S 13$. This value is then used for all simulations. 

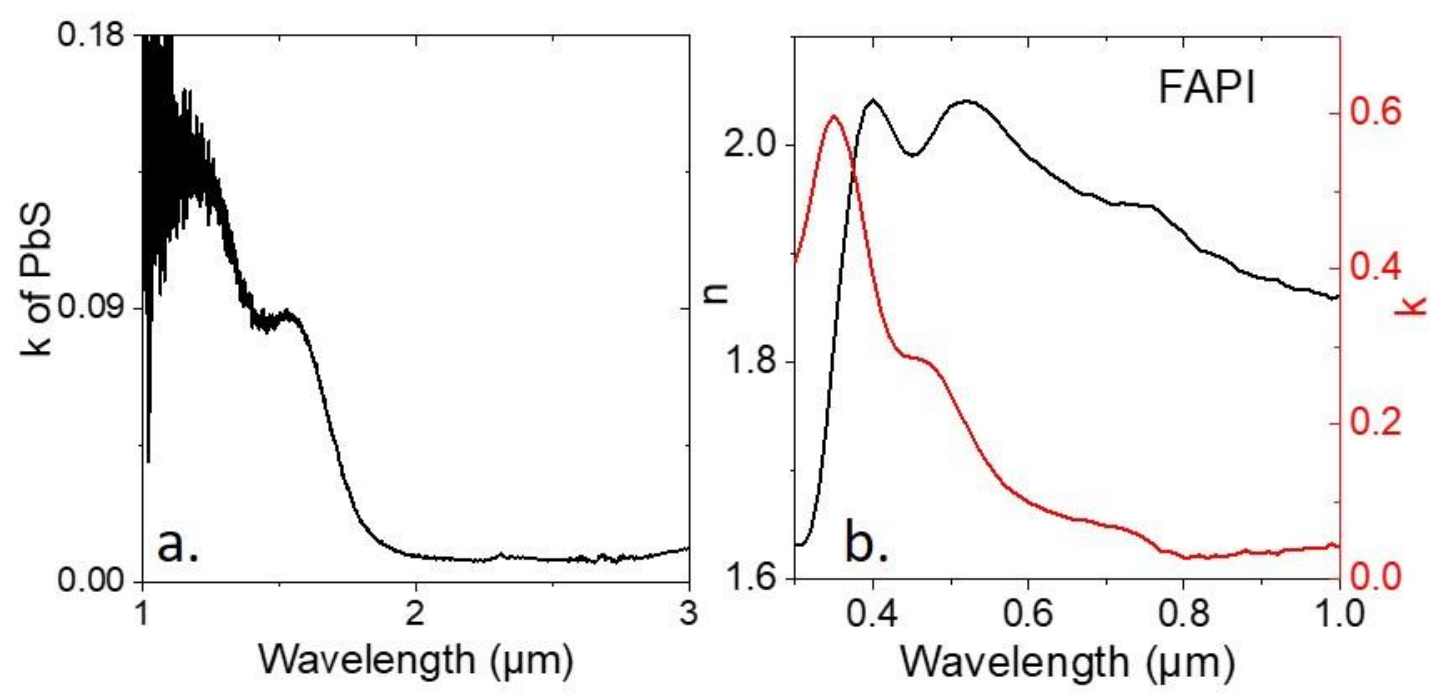

Figure S 12a. Imaginary part of the refractive index of PbS nanocrystals. $b$. Refractive index of FAPI measured by ellipsometry.

Table S3: Simulated parameters (period and gold width) for different refractive indices of the NCs film to induce a resonance in TM mode.

\begin{tabular}{ccc}
\hline \hline $\mathbf{n}$ & period $(\boldsymbol{\mu m})$ & $\mathbf{w}_{\mathbf{A u}}(\mathbf{n m})$ \\
\hline \hline 1.5 & 1.12 & 230 \\
\hline 1.6 & 1.09 & 250 \\
\hline 1.7 & 1.06 & 250 \\
\hline 1.8 & 1.03 & 270 \\
\hline 1.9 & 1.01 & 280 \\
\hline \hline
\end{tabular}

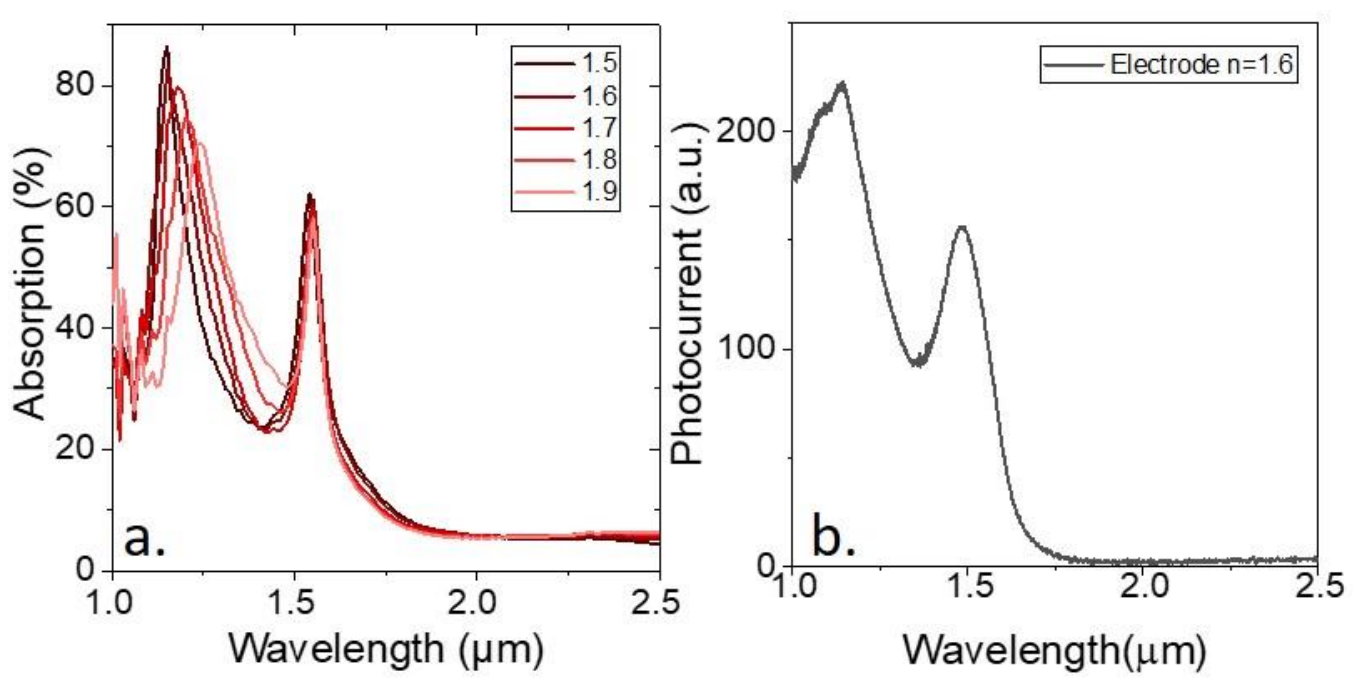

Figure S 13a. Simulated absorption of the GMR electrodes for different refractive index. $b$. Spectrum of the electrode corresponding to $n=1.6$. 


\subsection{Effect of the PbS content}
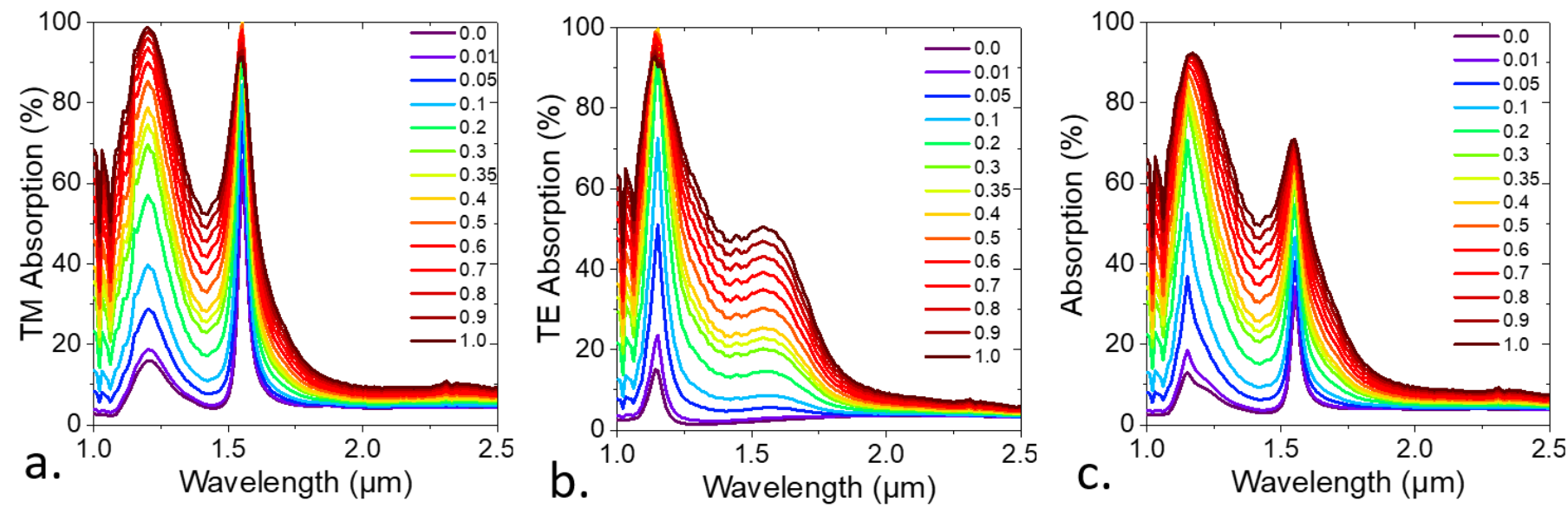

Figure S 14a. Simulated absorption spectra for the TM mode for various content of PbS in a FAPI/PbS mixture. (0 corresponds to pure FAPI). b. Simulated absorption spectra for the TE mode for various content of $\mathrm{PbS}$ in a FAPI/PbS mixture. c. Simulated absorption spectra for unpolarized light for various content of $P b S$ in a FAPI/PbS mixture.

\subsection{Dispersion relation}

The simulated dispersion relation for the GMR electrodes is provided in Figure S 15a (resp b.) for the TM mode (resp TE). In blue, the absorption is highlighted. White dashed lines correspond to the appearing of a diffracted order within the air. The white dot-dashed line corresponds to another GMR resonance.
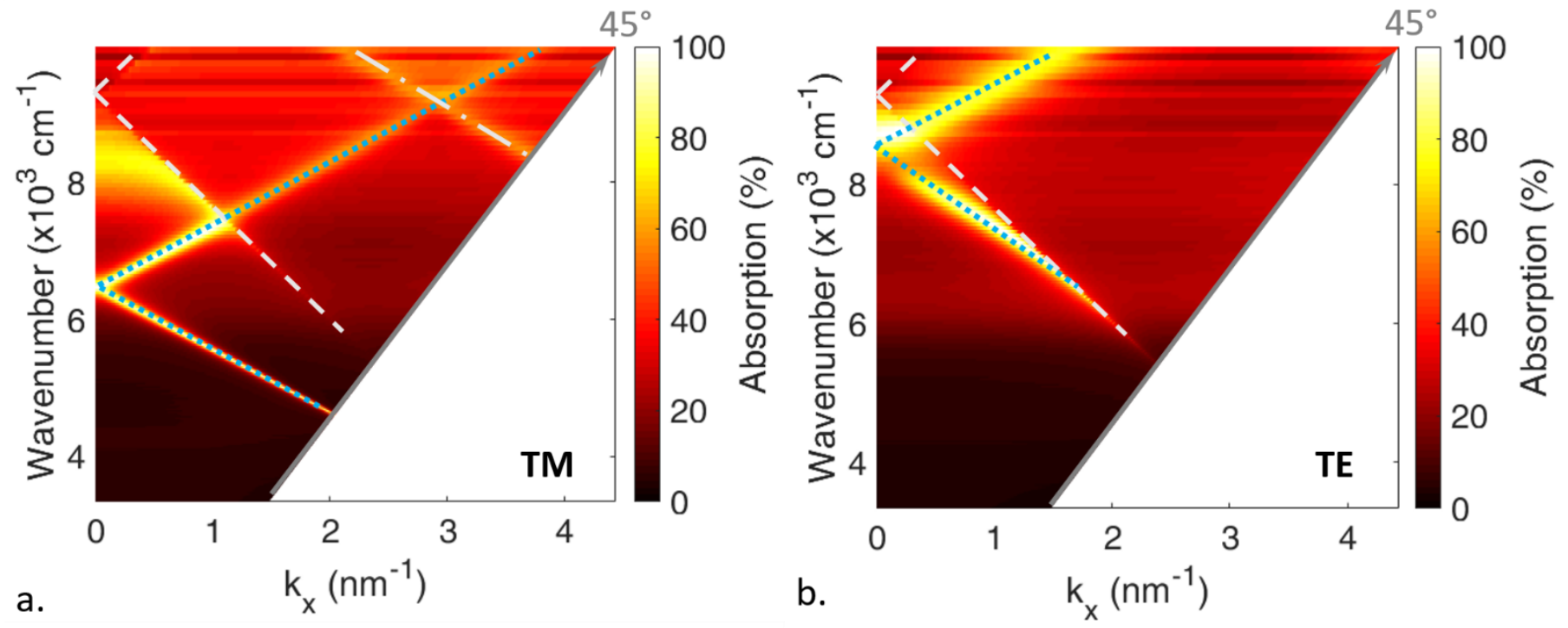

Figure S 15: a. (resp. b.) Angle-resolved specular TM (resp. TE) absorption simulation through interdigitated electrodes on a $\mathrm{Au} / \mathrm{SiO}_{2}$ substrate designed to induce GMRs, in linear color map as a function of $\sigma=1 / \lambda$, the wavenumber, and $k_{x}=2 \pi \cdot \sin (\theta) / \lambda$, the $x$ component of the incident wave vector.

\subsection{Electrodes fabrication}

The Figure $\mathbf{S} \mathbf{1 6}$ is a schematic of these main fabrication steps. 


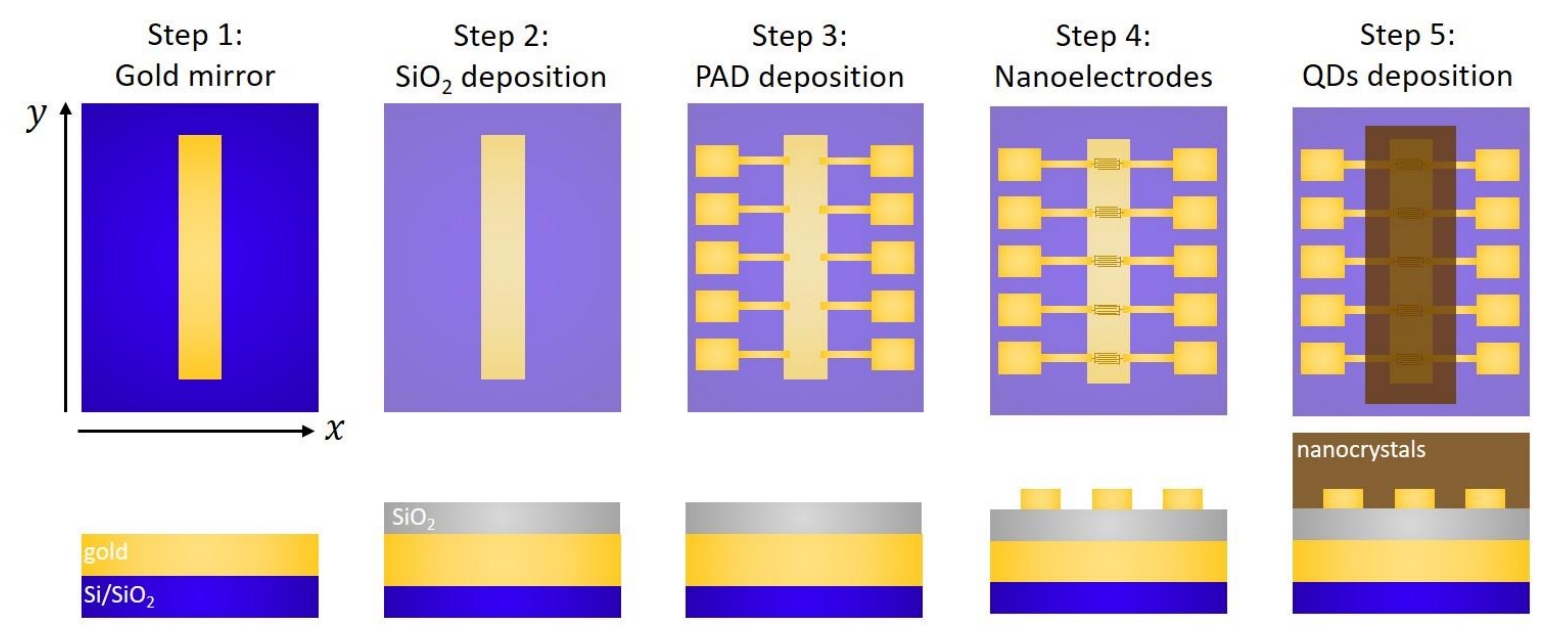

Figure S 16 Main fabrication steps of the electrodes used to induce a GMR. Top part is a top view, while bottom part is a side view. Step one relates to the deposition of the gold mirror. Step 2 relates to the deposistion of $\mathrm{SiO}_{2}$ dielectric to avoid shorts through the back mirror. Step 3 relates to the deposition of the macroscopic pads. Step 4 relates to the fabrication of the interdigitated electrodes inducing GMR, thanks to e-beam lithography. Step 5 relates to the CQD film deposition. Adapted with permission from ref 5. Copyright (2019) American Chemical Society.

Electrode inducing GMR. These electrodes are made using 4 different steps.

Step 1: Deposition of the gold mirror. The surface of $\mathrm{Si} / \mathrm{SiO}_{2}$ wafer ( $400 \mathrm{~nm}$ oxide layer) is cleaned by sonication in acetone. The wafer is rinsed with acetone, then isopropanol and dried with a $\mathrm{N}_{2}$ gun. A final cleaning is made using an $\mathrm{O}_{2}$ plasma. An adhesion promoter (TI PRIME) is spin-coated onto the substrate and baked at $120^{\circ} \mathrm{C}$ for $2 \mathrm{~min}$. AZ5214E is spin-coated and baked at $110^{\circ} \mathrm{C}$ for $90 \mathrm{~s}$. The substrate is exposed under UV through a pattern mask for $1.5 \mathrm{~s}$. The film is then baked at $125^{\circ} \mathrm{C}$ for 2 min in order to invert the resist. Then a $40 \mathrm{~s}$ flood exposure is performed. The resist is developed using a bath of AZ726 for $32 \mathrm{~s}$, rinsed in pure water and finally dried with $\mathrm{N}_{2}$. We then deposit $5 \mathrm{~nm}$ of chromium and $80 \mathrm{~nm}$ of gold using thermal evaporation. The lift-off is performed by dipping the film in acetone for 1 hour. The gold mirror is rinsed using isopropanol and dried with a $\mathrm{N}_{2}$ flow.

Step 2: Insulating gold mirror. The samples patterned with the gold mirror are put in an Alcatel sputtering chamber for a 45 min deposition of SiOx, at $120 \mathrm{~W}$. At the end of sputtering, the obtained SiOx layer is 120 $\mathrm{nm}$ thick.

Step 3 Deposition of macroscopic pads for reporting electric contacts. After having rinsed the sample with acetone and isopropanol, the adhesion promoter and the resist are deposited as described in step 1. The sample is then exposed through a second chromium mask for $1.5 \mathrm{~s}$. The same resist inversion process as described in step 1 (annealing + flood exposure) is performed before developing the sample in AZ726 developer for $25 \mathrm{~s}$ and rinsing it in pure water. The patterned sample is then introduced into a VINCI evaporator for depositions of $5 \mathrm{~nm} \mathrm{Cr}$ and $150 \mathrm{~nm} \mathrm{Au}$. At the end, the sample is dipped in acetone overnight to remove remaining resist.

Step 4 Deposition of nanoelectrodes. After having rinsed the sample with acetone and isopropanol, a layer of A6 PMMA 950 is spin-coated onto the substrate and baked for $15 \mathrm{~min}$ at $150{ }^{\circ} \mathrm{C}$. The samples are transferred in a Zeiss Supra 40 SEM with Raith elphy quantum device for electron beam lithography. The operating bias is set to $20 \mathrm{kV}$ and the aperture to $15 \mu \mathrm{m}$. The current is measure at $70 \mathrm{pA}$. The dose is set at $220 \mu \mathrm{C} . \mathrm{cm}^{-2}$. The PMMA is developed by dipping the film in a solution of MIBK:isopropanol (1:3) for $50 \mathrm{~s}$ and 
rinsed in pure isopropanol for $20 \mathrm{~s}$. We then deposit a $5 \mathrm{~nm}$ layer of chromium and a $50 \mathrm{~nm}$ layer of gold using thermal evaporation. The lift-off is performed by dipping the film in acetone overnight. Sonication at low power is necessary to remove the remaining resist. 20 pairs of digits are deposited as electrodes. The electrodes are $500 \mu \mathrm{m}$ long. For a resonance at $1.5 \mu \mathrm{m}$ the digits are $350 \mathrm{~nm}$ wide and separated by 750 $\mathrm{nm}$. For a $2.5 \mu \mathrm{m}$ resonance, the digits are $900 \mathrm{~nm}$ wide and separated by $900 \mathrm{~nm}$.

\subsection{Photoluminescence characterization of the GMR electrode}

To be able to measure luminescence, no ligand exchange is performed on the film. The photoluminescence is measured using an Olympus BX51WI upright microscope with wide field Köhler illumination for the optical pumping (light source: a tungsten incandescent bulb filtered with a Thorlabs FESH0800 short pass filter to remove the UV and infrared part of the spectrum). The 50X near-infrared objective used for optical pumping also serves to collect the photoluminescence. The latter is separated from the optical pump with a longpass dichroic mirror (Thorlabs DMLP950R filter) and imaged onto an InGaAs camera (Princeton Instruments NIRvana 640ST) with the help of a $20 \mathrm{~mm}$ Telan lens (an additional longpass colored glass filter is put in front of the camera to remove any remnant of visible light). To obtain Figure S 17c-d, a Bertrand lens is inserted in the optical path after the dichroic mirror to image the back focal plane of the microscope objective.

To verify an immediate effect of the GMR electrodes on the electromagnetic field, we have imaged the photoluminescence $(\mathrm{PL})$ signal of the field in the vicinity of the GMR electrodes using an upright optical microscope, see Figure S 17b. We observed a clear enhancement in the PL signal on the top of the GMR electrode, see Figure S 17a. By imaging the Fourier plane, we can observe the directionality of the PL signal induced by the resonator. In Figure S 17c, same image taken outside the GMR electrodes, we observe an isotropic intensity, while a clear directionality pattern is observed on top of the electrode, see Figure S 17d.
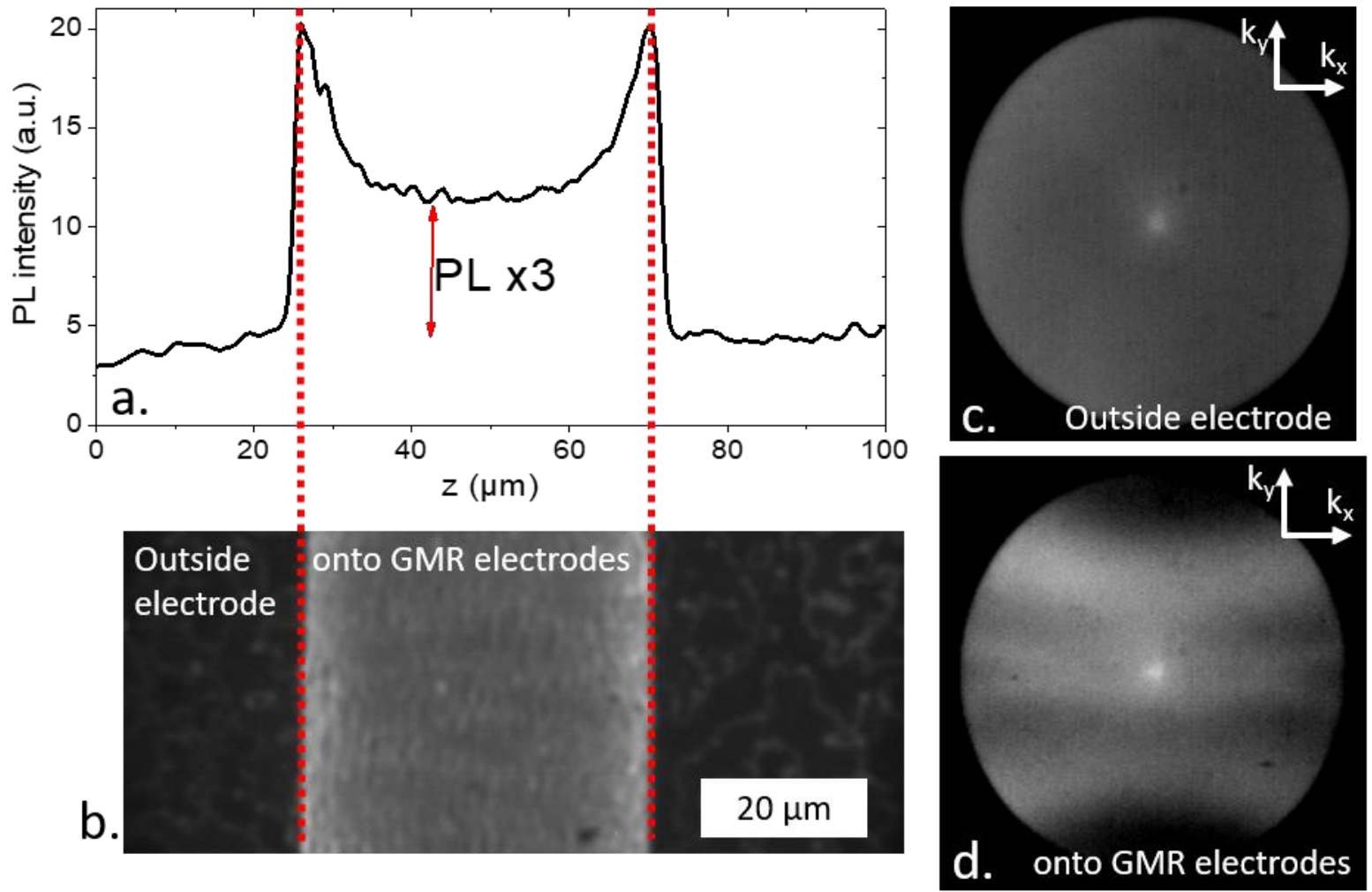

Figure S 17a. Intensity profile of the photoluminescence along the GMR electrode for a FAPI/PbS nanocrystal mixture with $35 \% \mathrm{PbS}$ content. $b$. Infrared image (visible part has been filtered) of the photoluminescence around the GMR electrode for a FAPI/PbS nanocrystal mixture with 35\% PbS content. c. (respectively d.) is an image of the back focal plane of the objective (Fourier imaging) giving the angular distribution of the luminescence signal outside (respectively onto) the GMR electrodes. The central spot on both images is an artefact of the experiment. 


\subsection{Additional characterization of the FAPI/PbS film deposited onto GMR electrode}

Figure $\mathrm{S} 18$ provide additional detector characterization (6-V curve, responsivity, noise, detectivity and time response) or the FAPI/PbS mixture deposited on the GMR electrodes, whilst the Table $\mathrm{S} 4$ compare the performance of the pristine PbS, with FAPI/PbS mixture and FAPI/PbS mixture deposited on GMR.
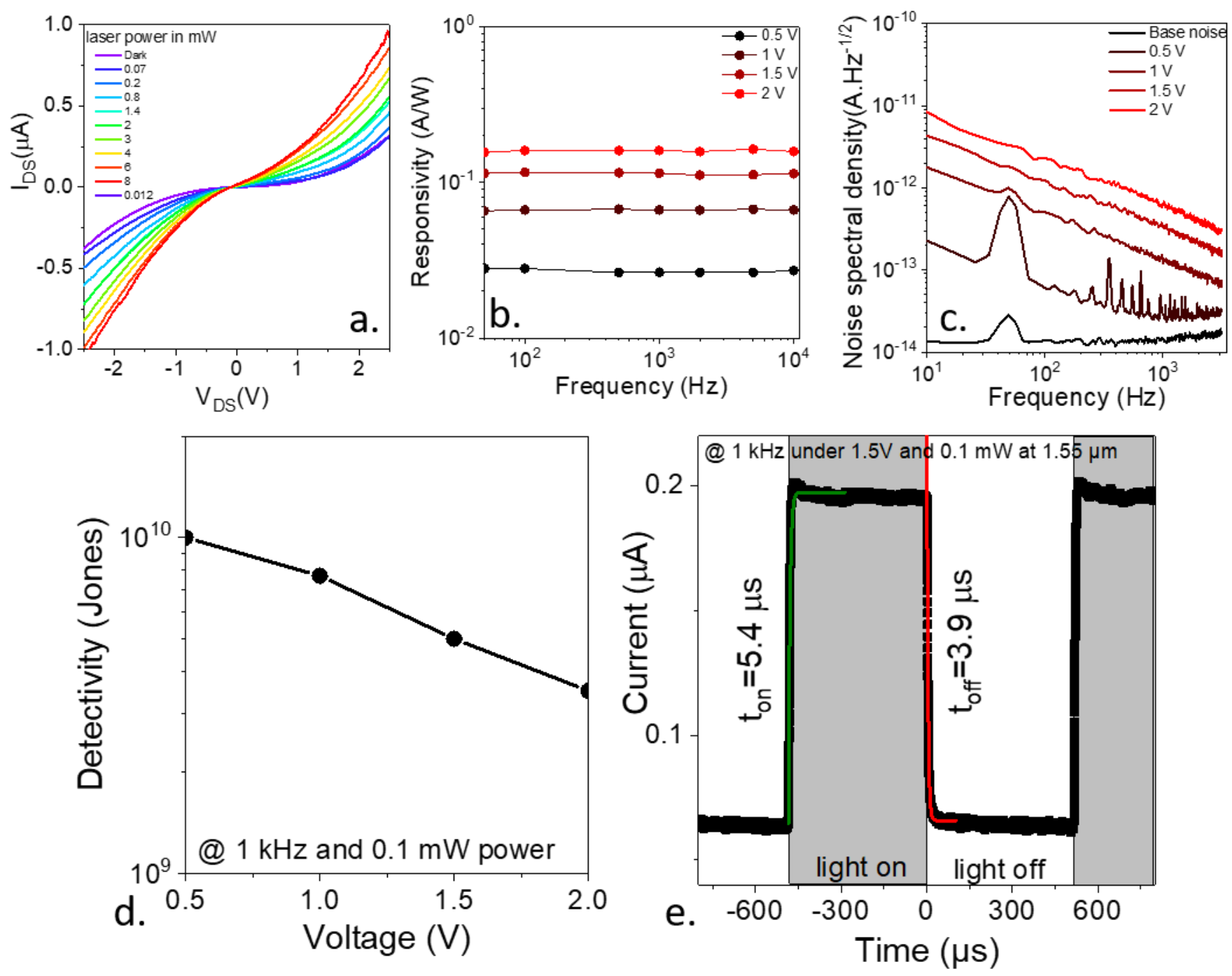

Figure S 18a. I-V curve for the FAPI/PbS (35\% PbS content) film deposited on the GMR electrode. Under illumination by a $1.55 \mu \mathrm{m}$ laser diode with various incident power. b. Responsivity of FAPI/PbS (35\% PbS content) film deposited on the GMR electrode as a function of the signal modulation. $c$. Noise current spectral density as a function of the signal frequency for FAPI/PbS (35\% PbS content) film deposited on the GMR electrode. $d$. Detectivity as a function of the applied bias for a FAPI/PbS mixture (35\% PbS content) onto GMR electrodes. e. Current as a function of time for a PbS/FAPI mixture (35\% PbS content) onto GMR electrodes as the light $\left(\lambda=1.55 \mu \mathrm{m}-P=0.03 \mathrm{~W} . \mathrm{cm}^{-2}\right)$ is turned on and off. 
Table 44 Infrared detection figures of merit at $1.55 \mu \mathrm{m}$ for pristine PbS, hybrid FAPI/PbS and hybrid FAPI/PbS coupled to GMR electrode.

\begin{tabular}{rcccc}
\hline \hline Material & $\begin{array}{c}\text { Responsivity } \\
\left(\mathbf{A} . \mathbf{W}^{-1}\right) \\
@ 0.1 \mathrm{~mW}, \mathbf{1 ~ k H z}\end{array}$ & $\begin{array}{c}\text { Noise }\left(\mathbf{A . H z} \mathbf{~}^{-1 / 2}\right) \\
@ 1 \mathbf{~ k H z}\end{array}$ & $\begin{array}{c}\text { Detectivity } \\
\text { (Jones) }\end{array}$ & $\begin{array}{c}\text { Decay time } \\
\text { response }(\mu \mathrm{s})\end{array}$ \\
\hline PbS @ 1 V & $3 \times 10^{-3}$ & $2 \times 10^{-12}$ & $7 \times 10^{8}$ & 200 \\
\hline FAPI/PbS (35\%) & $3.5 \times 10^{-4}$ & $5.4 \times 10^{-14}$ & $1 \times 10^{9}$ & 33 \\
\hline $\begin{array}{r}\text { FAPI/PbS (35\%) + } \\
\text { GMR }\end{array}$ & $3 \times 10^{-2}$ & $2.9 \times 10^{-14}$ & $1 \times 10^{10}$ & $<5$ \\
\hline \hline
\end{tabular}

\section{REFERENCES}

(1) Bergeard, N.; Silly, M. G.; Krizmancic, D.; Chauvet, C.; Guzzo, M.; Ricaud, J. P.; Izquierdo, M.; Stebel, L.; Pittana, P.; Sergo, R.; Cautero, G.; Dufour, G.; Rochet, F.; Sirotti, F. Time-Resolved Photoelectron Spectroscopy Using Synchrotron Radiation Time Structure. J. Synchrotron Radiat. 2011, 18, 245-250.

(2) Hugonin, J.-P.; Lalanne, P. Reticolo Software for Grating Analysis. Reticolo Software for Grating Analysis, Institut d'Optique, Orsay, France 2005. Institut d'Optique, Orsay, France. 2005.

(3) Olmon, R. L.; Slovick, B.; Johnson, T. W.; Shelton, D.; Oh, S.-H.; Boreman, G. D.; Raschke, M. B. Optical Dielectric Function of Gold. Phys. Rev. B 2012, 86, 235147.

(4) Moreels, I.; Lambert, K.; De Muynck, D.; Vanhaecke, F.; Poelman, D.; Martins, J. C.; Allan, G.; Hens, Z. Composition and Size-Dependent Extinction Coefficient of Colloidal PbSe Quantum Dots. Chem. Mater. 2007, 19, 6101-6106.

(5) Chu, A.; Gréboval, C.; Goubet, N.; Martinez, B.; Livache, C.; Qu, J.; Rastogi, P.; Bresciani, F. A.; Prado, Y.; Suffit, S.; Ithurria, S.; Vincent, G.; Lhuillier, E. Near Unity Absorption in Nanocrystal Based Short Wave Infrared Photodetectors Using Guided Mode Resonators. ACS Photonics 2019, 6, 25532561. 